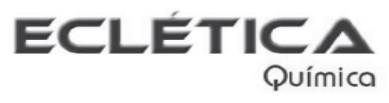

www.scielo.br/eq

Volume 29, número 2, 2004

\title{
Estudo da estabilidade térmica de óleos de peixes em atmosfera de nitrogênio
}

\author{
J.U. Garcia* ${ }^{*}$ H. I. dos Santos ${ }^{* *}$, A. P. Fialho ${ }^{* * *}$, F. L. T. Garro ${ }^{* * *}$, \\ N. R. Antoniosi Filho*, M. I. G. Leles* \\ * Instituto de Química - Universidade Federal de Goiás - UFG - 74001-970 - Goiânia - GO - Brasil. \\ ** Pró-Reitoria de Pesquisa e Pós-Graduação - Doutorando em Ciências Ambientais - UFG - 74001-970 - Goiânia - GO - Brasil. \\ *** Centro de Biologia Aquática - Universidade Católica de Goiás - UCG - 74605010 - Goiânia - GO - Brasil.
}

\begin{abstract}
Resumo: Os peixes são uma fonte rica em ácidos graxos poliinsaturados ômega 3 e esses ácidos fornecem benefícios para a saúde humana além da nutrição básica. Por essa razão muitos estudos têm sido feitos enfocando a análise do teor das substâncias que compõem os alimentos e também aos efeitos dos processamentos a que esses alimentos são submetidos. Esses estudos têm se estendido aos recursos pesqueiros. Vários estudos sobre a estabilidade térmica e/ou oxidativa e sobre o comportamento cinético de óleos vegetais têm sido realizados por Termogravimetria (TG), Análise Térmica Diferencial (DTA) e Calorimetria Exploratória Diferencial (DSC). No entanto, ainda são escassos os estudos sobre o comportamento térmico de óleos e gorduras de origem animal. Assim, avaliou-se a estabilidade térmica de cinco espécies de peixes do rio Araguaia (Goiás - Brasil) em atmosfera de nitrogênio por TG/DTG. Os resultados mostraram perdas de massa entre $235^{\circ} \mathrm{C}$ e $490^{\circ} \mathrm{C}$ e apontaram uma equivalência de comportamento termogravimétrico entre os óleos das cinco espécies e também entre esses óleos e alguns óleos vegetais, tais como, os óleos de araticum, buriti e dendê.
\end{abstract}

Palavras-chave: Óleos de peixes; estabilidade térmica; termogravimetria.

\section{Introdução}

Nos últimos anos muitas pesquisas têm sido realizadas com o objetivo de fornecer subsídios para uma análise da qualidade dos alimentos. Muitas delas são direcionadas ao estudo sobre os efeitos da temperatura de processamento a que esses alimentos são submetidos e outras à análise do teor das substâncias que os compõem [1, 3, 8, $9,13,14,25]$. Os estudos enfocando a análise do teor das substâncias que compõem os alimentos têm se estendido aos recursos pesqueiros, pois é sabido que os peixes são uma fonte rica em ácidos graxos poliinsaturados ômega 3 e que esses ácidos fornecem benefícios para a saúde humana além da nutrição básica. [4, 5, 6, 22, 27]
Vários estudos sobre a estabilidade térmica e/ou oxidativa e sobre o comportamento cinético de óleos também têm sido realizados, essencialmente de óleos vegetais e essenciais. Esses estudos têm sido realizados por Termogravimetria (TG), Análise Térmica Diferencial (DTA) e Calorimetria Exploratória Diferencial (DSC). [10, 15, 18]

Rudnid et al. [19] fizeram um estudo comparativo da estabilidade oxidativa do óleo de linhaça. Kowalski[11] estudou a decomposição térmica-oxidativa de óleos comestíveis e gorduras. Wesolowski e Erecinska[26] avaliaram a qualidade do óleo de sementes de colza usando análise térmica. Pereira e Das[17] usaram a técnica 
de Calorimetria Exploratória Diferencial (DSC) para estudar os efeitos de flavonóides na autooxidação térmica de óleos vegetais. Litwinienko e Kasprzycka-Guttman[12], também usando DSC, estudaram a cinética da oxidação térmica de óleos de mostarda. Tan e Che Man[23] monitoraram a oxidação de óleos aquecidos também usando DSC. Shen e Alexander[21] fizeram a análise térmica de ácidos graxos de cadeia longa. Santos et al.[20] determinaram o calor específico de alguns óleos comestíveis comerciais usando DSC. Um dos mais recentes estudos foi realizado por Faria et al.,[7] no qual foi avaliada a estabilidade térmica de óleos e gorduras de vegetais do cerrado, usando TG, DTG e DTA. As técnicas termoanalíticas utilizadas nesses trabalhos forneceram dados sobre a estabilidade térmica e geraram subsídios para análise da qualidade dos mesmos. No entanto, ainda são escassos os estudos sobre o comportamento térmico de óleos e gorduras de origem animal.

Por conseguinte, realiza-se neste trabalho o estudo termogravimétrico de óleos de peixes coletados no rio Araguaia, rio este que corresponde ao um dos mais importantes recursos hídricos das regiões Centro-Oeste e Norte do Brasil.

\section{Materiais e métodos}

\section{Amostragem}

Para esse estudo foram coletadas e identificadas cinco espécies de peixes do Rio Araguaia, no município de São Miguel do Araguaia, localizado no norte de Goiás, nos dias 15 e 16 de novembro de 2002. As espécies Cichla ocellaris (tucunaré), Metynnis hypsauchen (pacu prateado), Mylossoma duriventre (pacu manteiga), Osteoglossum bicirrhosum (aruanã) e Pygocentrus nattereri (piranha caju) foram fotografadas e classificadas pelo Centro de Biologia Aquática da Universidade Católica de Goiás.

A extração de óleos de peixes foi realizada a partir de modificações do método descrito por Duarte[3] como descrito a seguir:

Retirou-se os filés de três peixes de cada espécie. Triturou-se esses filés usando um liquidificador e, então, pesou-se $50 \mathrm{~g}$ do tecido triturado. Em seguida, transferiu-se essa massa para uma cápsula de porcelana que foi levada a aquecimento em estufa a uma temperatura de $100^{\circ} \mathrm{C}$ até a obtenção de massa constante (um período de aproximadamente oito horas). O tecido seco foi então transferido para um erlenmeyer de $500 \mathrm{~mL}$, no qual foram adicionados $10 \mathrm{~g}$ de sulfato de sódio anidro e $200 \mathrm{~mL}$ de hexano, promovendose a agitação do sistema por uma hora. Em seguida foram realizadas a filtração e evaporação do solvente em rotaevaporador.

Os óleos obtidos foram acondicionados em frascos âmbar sob atmosfera de nitrogênio.

\section{Análise Térmica (TG/DTG)}

As curvas termogravimétricas foram obtidas em um equipamento do modelo TGA/ SDTA851 ${ }^{\mathrm{e}}$ da marca Mettler Toledo. O intervalo de temperatura explorado foi de 25 a $600^{\circ} \mathrm{C}$ e a razão de aquecimento de $10^{\circ} \mathrm{C} \mathrm{min}{ }^{-1}$. Foi utilizada uma atmosfera dinâmica de nitrogênio a uma vazão de $50 \mathrm{~mL} \mathrm{~min}{ }^{-1}$. Utilizou-se um cadinho de a-alumina e massas iniciais das amostras variando de 13,9 a 17,6 mg, aproximadamente.

\section{Resultados e discussão}

Na Figura 1 são apresentadas as curvas TG em atmosfera dinâmica de nitrogênio dos óleos das cinco espécies de peixes analisadas.

As curvas termogravimétricas dos óleos de peixes analisados apresentaram perfis semelhantes com perda de massa $(93,3$ a 99,1\%) em uma única etapa, entre 290 e $490^{\circ} \mathrm{C}$, relativas à volatilização e/ou decomposição do óleo. As amostras analisadas apresentaram temperatura final de decomposição entre 474 e $490^{\circ} \mathrm{C}$, como mostrado na Figura 2. Essa semelhança nos perfis das curvas se deve à similaridade entre as composições de ácidos graxos dos óleos de peixes dessa região, que apresentam majoritariamente os ácidos palmítico (16:0), oléico (18:1w9), linoléico (18:2w6), palmitoleico (16:1w9) e esteárico (18:0), e um teor de ácidos graxos insaturados por volta de $48 \% .3$

Observa-se, no intervalo de temperaturas de 30 até a temperatura de início da primeira etapa de perda de massa, uma perda de massa contínua e de pequena intensidade. Esta perda de massa corresponde em percentagem de perda a 0,9\% para o óleo de aruanã, 6,7\% para o óleo de pacu manteiga, 6,1\% para o óleo de pacu prateado, 3,1\% 
para o óleo de piranha caju e 2,8\% para o óleo de tucunaré, provavelmente devido à volatilização de resíduos de hexano remanescente da extração dos óleos de peixe e ácidos graxos livres presentes em pequenas quantidades em amostras lipídicas desta natureza, tais como os ácidos palmítico, palmitoleico e esteárico. A Figura 3 mostra a curva termogravimétrica do ácido esteárico e evidencia que ácidos graxos livres sofrem decomposição em temperaturas inferiores as temperaturas de estabilidade dos óleos.

A variação do valor da massa inicial das amostras dos diferentes óleos, $\left(13,9 £ \mathrm{~m}_{\mathrm{i}} £ 17,6\right)$ $\mathrm{mg}$, ocorre em razão da dificuldade de se adicionar ao cadinho uma mesma massa de amostras nessa natureza. No entanto, foi observado que essa variação não afeta os intervalos de perda de massa e não influi nos resultados mostrados na Figura 2. Isso foi verificado variando-se a massa inicial de duas amostras de um mesmo óleo. A Figura 4 mostra duas curvas TG de duas amostras do óleo de tucunaré, uma com massa inicial de 8,4 mg e a outra com massa inicial de 15,8 mg. Observa-se que as duas amostras apresentam perda de massa no mesmo intervalo de temperatura.

Verifica-se que as faixas de temperatura de estabilidade e de temperatura final de decomposição (Figura 2) são próximas àquelas encontradas em óleos vegetais e superiores as obtidas para gorduras vegetais (Tabela 1)[7], as quais possuem maior teor de triacilglicerídeos contendo ácidos graxos de cadeia curta, os quais são mais voláteis que os triacilglicerídeos de cadeia longa. A Figura 5 mostra as curvas TG da gordura de babaçu e do óleo de dendê. A gordura de babaçu apresenta um maior teor de triacilglicerídeos contendo ácidos graxos com menor comprimento de cadeia16 e, por isso, sua volatilização se inicia e termina em temperaturas inferiores às temperaturas de início e final de volatilização do óleo de dendê, apesar de o óleo de dendê apresentar um maior teor de ácidos graxos insaturados[24] e de estes serem mais instáveis e voláteis que os ácidos graxos saturados.

\begin{tabular}{|l|c|c|}
\hline \multicolumn{1}{|c|}{ Óleo } & $\begin{array}{c}\text { Temperatura de } \\
\text { estabilidade } /{ }^{\circ} \mathrm{C}\end{array}$ & $\begin{array}{c}\text { Temperatura final } \\
\text { da decomposição } /{ }^{\circ} \mathrm{C}\end{array}$ \\
\hline Araticum & 320 & 478 \\
\hline Babaçu & 180 & 440 \\
\hline Buriti & 321 & 483 \\
\hline Guariroba & 283 & 433 \\
\hline Murici & 271 & 477 \\
\hline
\end{tabular}

Tabela 1 - Temperatura de estabilidade e temperatura final da decomposição obtida das curvas TG/DTG dos óleos vegetais em atmosfera de nitrogênio.

Fonte: Referência 7

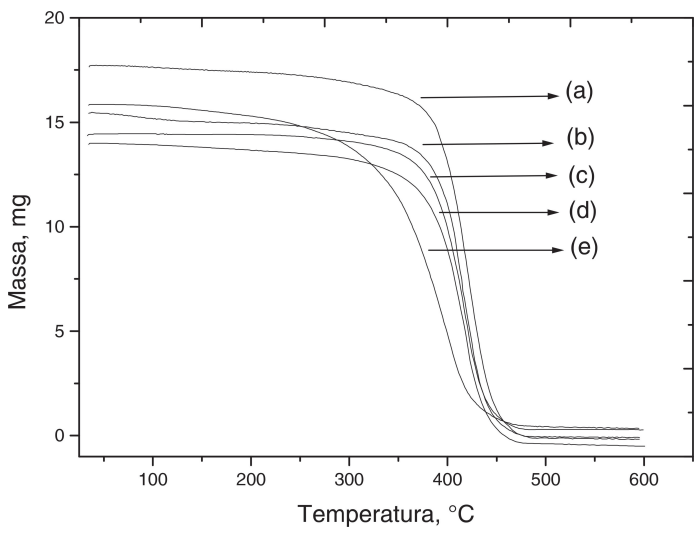

Figura 1 - Curvas TG em atmosfera de nitrogênio dos óleos de: (a) pacu prateado $(\mathrm{mi}=17,6 \mathrm{mg})(\mathrm{b})$ pacu manteiga $(\mathrm{mi}=$ $15,4 \mathrm{mg})$ (c) piranha caju $(\mathrm{mi}=14,4 \mathrm{mg})(\mathrm{d})$ aruanã $(\mathrm{mi}=$ $13,4 \mathrm{mg})(\mathrm{e})$ tucunaré $(\mathrm{mi}=15,8 \mathrm{mg})$.

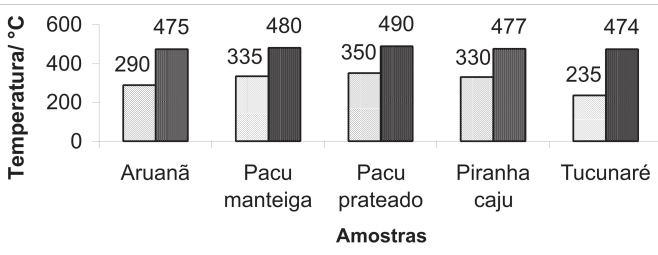

口Temperatura de estabilidade $\square$ Temperatura final de decomposição

Figura 2 - Temperatura de estabilidade térmica e temperatura final de volatilização e/ou decomposição de óleos de peixes em atmosfera de nitrogênio.

Assim, a partir das observações feitas por Faria et al.[7] e dos resultados mostrados na Figura 5, é possível correlacionar as temperaturas inicial e final de decomposição e/ou volatilização com o tamanho e o grau de insaturação dos triacilglicerídeos presentes nessas amostras. Neste sentido, a partir dos dados de temperatura inicial e final de volatilização e/ou decomposição de óleos de peixes em atmosfera de nitrogênio, apresentados na Figura 2, é possível estimar que 
o óleo de tucunaré, por apresentar menor temperatura de estabilidade térmica $\left(\mathrm{T}_{\mathrm{i}}=235^{\circ} \mathrm{C}\right)$, apresenta maior teor de triacilglicerídeos de cadeia curta que o óleo de aruanã $\left(\mathrm{T}_{\mathrm{i}}=290^{\circ} \mathrm{C}\right)$, seguidos dos óleos de piranha caju $\left(\mathrm{T}_{\mathrm{i}}=330^{\circ} \mathrm{C}\right)$, pacu manteiga $\left(\mathrm{T}_{\mathrm{i}}=335^{\circ} \mathrm{C}\right)$ e pacu prateado $\left(\mathrm{T}_{\mathrm{i}}=350\right.$ $\left.{ }^{\circ} \mathrm{C}\right)$.

O óleo de pacu prateado, por apresentar maior temperatura de estabilidade térmica $\left(\mathrm{T}_{\mathrm{i}}=350\right.$ ${ }^{\circ} \mathrm{C}$ ), deve apresentar menor teor de triacilglicerídeos de cadeia curta e maior teor de triacilglicerídeos de cadeia longa, o que é confirmado pelo fato de apresentar maior temperatura final de estabilidade térmica $\left(\mathrm{T}_{\mathrm{f}}=490\right.$ $\left.{ }^{\circ} \mathrm{C}\right)$.

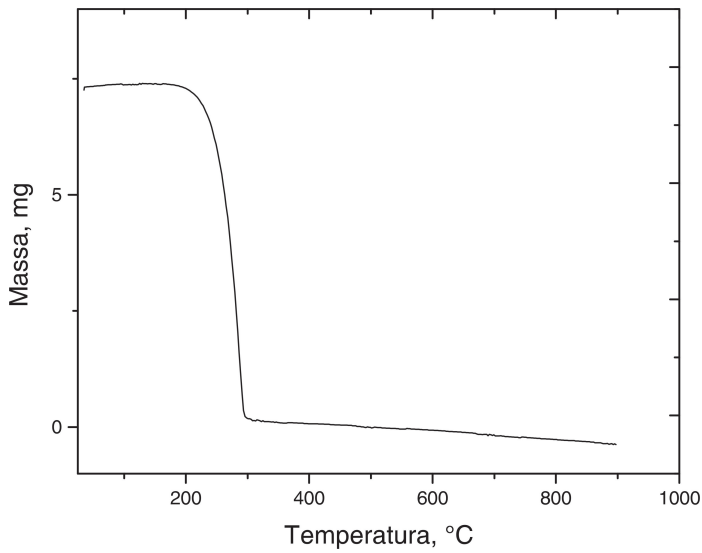

Figura 3 - Curva TG/DTG em atmosfera de nitrogênio do ácido esteárico ( $\mathrm{mi}=7,3 \mathrm{mg})$.

Desse modo, a análise termogravimétrica permite sugerir, de maneira relativa, o comprimento da cadeia do triacilglicerídeo presente num determinado óleo de peixe em comparação a outrem, o que seria difícil de ser determinado diretamente por Cromatografia Líquida de Alta Eficiência (HPLC). Tal dificuldade tem origem na complexidade das amostras de óleo de peixe, sendo que, mesmo a utilização da Cromatografia Gasosa Capilar a Altas Temperaturas (HT-CGC), a qual permite a separação dos triacilglicerídeos pelo comprimento da cadeia e grau de insaturação[2], seria, também, comprometida considerando o fato de que os triacilglicerídeos precisariam ser eluídos em temperaturas superiores a $450^{\circ} \mathrm{C}$ (estabilidade da maioria das fases estacionárias) como demonstrado a partir das curvas TG (Figuras 1).

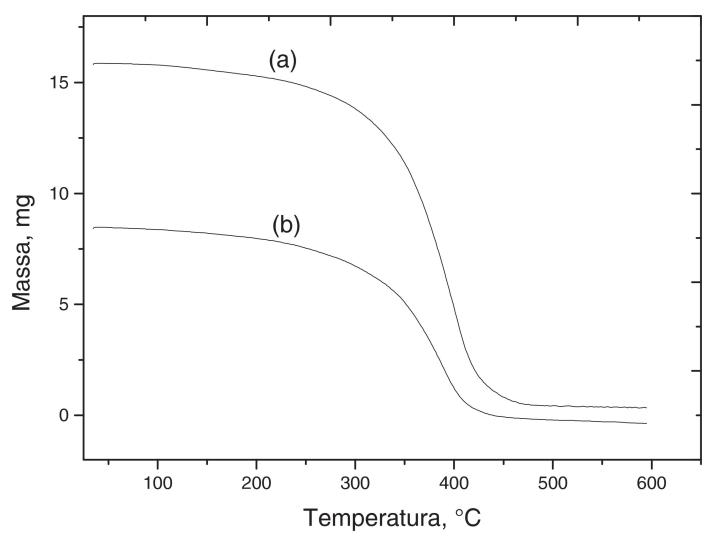

Figura 4 - Curvas TG de duas amostras de óleo de tucunaré de massas: (a) 15,8 mg (b) 8,4 mg (b).

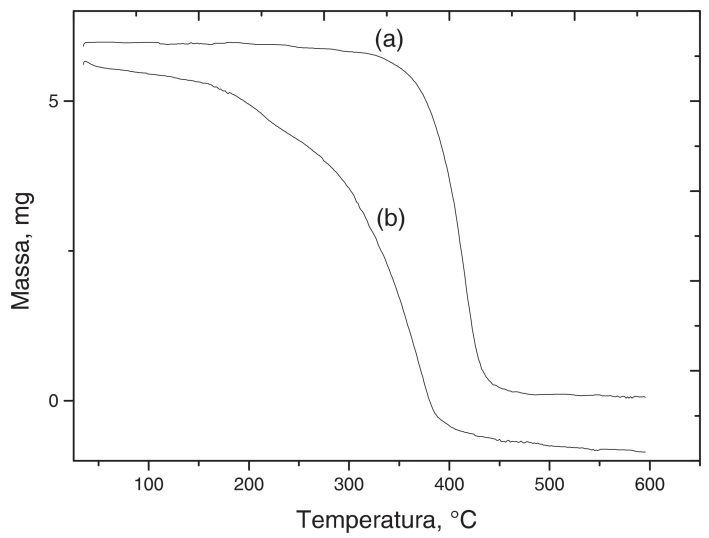

Figura 5 - Curvas TG: (a) óleo de dendê (mi = 5,9 mg) (b) gordura de babaçu ( $\mathrm{mi}=5,6 \mathrm{mg})$.

\section{Conclusão}

Os resultados obtidos por TG em atmosfera inerte, de nitrogênio, mostraram que o comportamento térmico dos óleos de peixes assemelha-se ao comportamento térmico observado para óleos vegetais, tais como, os óleos de araticum, buriti e dendê.

Todos os óleos tiveram perdas de massa em uma única etapa com uma variação de 93,3\% a $99,1 \%$, sofreram completa volatilização e/ou decomposição entre $235^{\circ} \mathrm{C}$ e $490^{\circ} \mathrm{C}$ e apresentaram a seguinte ordem crescente de estabilidade: óleo do tucunaré < óleo do aruanã < óleo de piranha caju $<$ óleo do pacu manteiga $<$ óleo de pacu prateado. 
Os resultados obtidos a partir da análise termogravimétrica permitem sugerir a técnica como uma ferramenta importante para a investigação do efeito da presença de triacilglicerídeos sobre a temperatura de estabilidade térmica de óleo de peixes, permitindo correlacionar o efeito com o tamanho relativo da cadeia do triacilglicerídeo (cadeia curta ou longa).

\section{Agradecimentos}

Os autores agradecem à Secretaria do Meio Ambiente e dos Recursos Hídricos do Estado de Goiás (SEMARH-GO) pelo apoio durante a coleta e ao Centro de Biologia Aquática da Universidade Católica de Goiás pela identificação dos peixes.

Recebido em 11/02/04

Aceito em 27/09/04

J. U. Garcia H. I. dos Santos A. P. Fialho F. L. T. Garro N. R. Antoniosi Filho M. I. G. Leles. Study of the thermal stability of fish oils under nitrogen atmosphere.

\begin{abstract}
The fish are a rich source of omega 3 polyunsaturated fatty acids that give benefits to human health beyond basic nutrition. For this reason a lot of studies have been done focusing on the analysis of the content of the substances that compose the foods and the processing effects on foods. These studies have been spread to fishing resources. Many studies about thermal oxidative stability and the kinetic behavior of vegetal oils have been made by Thermogravimetry (TG), Differential Thermal Analysis (DTA) and Differential Scanning Calorimetry (DSC). However, the studies about the oil and fat (from animals) thermal behavior are still lacking. Therefore, on this research analysed the thermal stability of five fish species from Araguaia river (Goiás - Brazil) in a nitrogen atmosphere by TG/DTG. The results showed losses of mass between $235^{\circ} \mathrm{C}$ and $490^{\circ} \mathrm{C}$ and it points to an equivalence of thermogravimetric behavior between the oils of the five fish species and also between the fish oils and some vegetal oils, such as, araticum, Brazilian wine-palm and palm oils.
\end{abstract}

Keywords: Fish oils; thermal stability; thermogravimetry.

\section{Referências}

[1] A. D. Andrade, et al. J. Am. Oil Chem. Soc. 72 (10) (1995) 1207.

[2] N. R. Antoniosi Filho, O. L. Mendes, F. M. Lanças, Chomatogr. 40(9-10) (1995) 557.

[3] G. R. M. Duarte, Estudo da composição de ácidos graxos e colesterol em óleos de peixes do Rio Araguaia. Goiânia, 2001. 98p. Dissertação (Mestrado) - Instituto de Química, Universidade Federal de Goiás.

[4] J. Dyerberg, H. O. Bang, Lanc. 3 (1979) 433.

[5] J. Dyerberg, H. O. Bang, Acta Med. Scand.192 (1972) 85.

[6] J. Dyerberg, Nutr. Res. 44(4) (1986) 125.

[7] E. A. de Faria, et al. Eclét. Quím. 27 (2002) 111.

[8] A. A. Gonçalves, C. Prentice-Hernández, Ciênc. Tecnol. Aliment.18 (1998) 4.

[9] R. S. de Jesus, E Lessi, A. Tenuta-Filho, Ciênc.

Tecnol. Aliment. 21(4) (2001) 144.
[10] E. Kaisersberger, Termochim. Acta 151 (1989) 81.

[11] B. Kowalski, Thermochim. Acta 184 (1991) 49.

[12] G. Litwinienko, T. Kasprzycka-Guttman, Thermochim. Acta 319 (1998) 185.

[13] E. L. Maia et al. Prochilodus cearensis. Ciênc. Tecnol. Aliment., v. 19, (3), 1999.

[14] E. L. Maia, Otimização da metodologia para caracterização de constituintes lipídicos e determinação da composição em ácidos graxos e aminoácidos de peixes de água doce. Campinas, 1992, 242p. Tese (Doutorado) - Faculdade de Engenharia de Alimentos da Universidade Estadual de Campinas, Universidade Estadual de Campinas.

[15] R. J., Ochocka, M., Wesolowski, H. Lamparczyk, Termochim. Acta 173 (1990) 199. 
[16] A. L. de Oliveira, L. A. Gioielli, M. N. Oliveira, Ciênc. Tecnol. Aliment., Campinas, 19(2) (1999) 270.

[17] T. A. Pereira, N. P. Das, Thermochim. Acta 165 (1990) 129.

[18] F.J., Rey, O., Chamorro, F. J. M., Gil, J. M.

Gil, J. Therm. Anal. v.40, 463, 1993.

[19] Rudnit et al., Thermochim. Acta 370 (2001) 135.

[20] J. C. de O. Santos et al. Calor específico de alguns óleos comestíveis comerciais por DSC. $1^{\text {st }}$ Pan-American and $2^{\text {nd }}$ Brazilian Congress on Thermal Analysis and Calorimetry, abr 2000.
[21] L. Shen, K. S. Alexander, Thermochim. Acta, 340-341 (1999) 271.

[22] A. P. Simopoulos, Canad. J. Physiol. Pharmacol. 75(3) (1997) 234.

[23] C. P. Tan, Y. B. Che Man, Food Chem. 67 (1999) 177.

[24] J.A. Trujillo-Quijano, W. Esteves, G.F. Plonis, D.B. Rodriguez-Amaya, Ciênc. Tecnol. Aliment. 12(1) 1992) 91.

[25] J. V. Visentainer et al. Ciênc. Tecnol. Aliment. 20(1) (2000) 90.

[26] M., Wesolowski, J. Erecinska. Thermochim. Acta 323 (1998) 137.

[27] T. Yamada et al., Atheroscler 153 (2000) 469. 